\title{
Formadores de didáctica de las ciencias experimentales y matemática: El self-studycolaborativo interinstitucional como motor de transformación de la docencia universitaria
}

\author{
Carlos Mario Vanegas Ortega 1 (1) @ \\ Carolina Paz Martínez Galaz ${ }^{2}$ (1) @ \\ Carolina Andrea Henríquez Rivas ${ }^{2}$ (D) @ \\ Carla Hernández Silva ${ }^{1}$ (D) @ \\ ${ }^{1}$ Universidad de Santiago de Chile; ${ }^{2}$ Universidad de Talca, Chile.
}

Resumen. En el marco de los desafíos profesionales que plantea hoy la docencia universitaria, en este artículo caracterizamos los procesos reflexivos que vivenciamos didactas de las ciencias experimentales y de la matemática, cuando desarrollamos un self-study colaborativo interinstitucional. Para ello, hemos adaptado el ciclo deweyniano según dos procesos reflexivos: el primero, de autoconfrontación con lo que ocurre en nuestras clases universitarias y lo que plantean los estudiantes mediante tickets de salida, y el segundo, de resignificación de la docencia universitaria con los amigos críticos. Los resultados muestran que cada didacta experimentó distintos procesos de reflexión en cuanto a las dimensiones relacionadas con los estudiantes, el contexto, la didáctica específica y la profesionalidad; además, demostramos que el conocimiento didáctico permite la configuración de procesos reflexivos e identitarios diferenciadores que resultan claves en la comprensión y resignificación de las prácticas pedagógicas.

Palabras clave: self-study; formación de profesores; reflexión; didáctica de las ciencias; didáctica de las matemáticas.

\begin{abstract}
Formadores em didática das ciências experimentais e matemática: 0 self-study colaborativo interinstitucional como motor de transformação da docência universitária

Resumo. No âmbito dos desafios profissionais propostos pela docência universitária hoje, caracterizamos neste artigo os processos de reflexão vivenciados pelos didatas das ciências experimentais e da matemática ao realizarem um self-study colaborativo interinstitucional. Para isso, adaptamos o ciclo de Dewey de acordo com dois processos de reflexão: o primeiro, de autoconfrontação com o que acontece nas nossas aulas na universidade e o que os estudantes propõem mediante bilhetes de saída; e o segundo, de ressignificação da docência universitária com os amigos críticos. Os resultados mostram que cada didata experimentou diferentes processos de reflexão quanto às dimensões relacionadas com os estudantes, o contexto, a didática específica e o profissionalismo; além disso, demostramos que o conhecimento didático permite a configuração de processos de reflexão e identitários diferenciadores que são fundamentais na compreensão e ressignificação das práticas pedagógicas.
\end{abstract}

Palavras-chave: self-study, formação de professores, reflexão, didática das ciências, didática da matemática:

Trainers in didactic of sciences and mathematics: The inter-institutional collaborative self-study as an engine of transformation of university teaching

Abstract. Within the framework of the professional challenges posed by university teaching today, in this article we characterize the reflexive processes that we live as didactics of experimental sciences and mathematics, when we develop an inter-institutional collaborative self-study. For this, we have adapted the Deweynian cycle according to two reflexive processes: the first, of self-confrontation about what happens in our university classes in contrast to the points made by students using tickets out, and the second, of resignification of university teaching with critics friends. The results show that each didact underwent different processes of reflection regarding the dimensions related to the students, the context, the specific teaching and professionalism. In addition, we demonstrate that didactic knowledge allows the configuration of differentiating reflective and identity processes that are key in the understanding and resignification of pedagogical practices.

Keywords: self-study; teacher training; teacher reflection; science teaching; mathematics teaching 


\section{Introducción}

En los últimos veinte años, la formación inicial y continua de profesores ha realizado esfuerzos por incorporar de manera intencionada el conocimiento didáctico como un eje fundamental del quehacer profesional docente (Guzmán, 2011; Porlán, 2018; Sanmartí \& Marchán, 2015). Sin embargo, en Chile, ha sido lento el proceso de aceptación del conocimiento didáctico del contenido (también llamado CDC o PCK/MTSK por sus siglas en inglés) (Vergara \& Cofré, 2014). De hecho, sólo desde el año 2016 y en el marco de diversos planes de mejoramiento institucional (PMI) financiados por el Ministerio de Educación para la formación de profesores, se han podido incorporar especialistas en didácticas específicas al cuerpo académico.

En ese sentido, quienes nos hemos dedicado a la didáctica de las ciencias y la matemática en la educación superior, coincidimos en que hemos pasado por tres etapas de desarrollo profesional docente:

- Etapa I: Formación. Ocurrió en nuestros programas de pregrado y posgrado, desde distintas perspectivas epistemológicas, pero con el mismo interés: desarrollar un conocimiento profesionalizante de la enseñanza que sea propio del profesorado.

- Etapa II: Instalación. Acontece con la inserción laboral a una casa de estudios dedicada a la formación de profesores. Por lo general, se nos ve como profesores con formación científica que tenemos 'algo más' que aportar, lo que se traduce en hacernos cargo de la docencia en áreas científicas, prácticas pedagógicas, evaluación, currículo, trabajos de graduación, y en el mejor de los casos, algunas asignaturas de didáctica.

- Etapa III: Validación. Corresponde al conjunto de tensiones que experimentamos a diario para dar relevancia al conocimiento didáctico frente al conocimiento científico (biología, física, química o matemática). Esto implica ganar más espacios curriculares para la reflexión y el análisis didáctico, lograr que los futuros profesores hagan trabajos de graduación asociados a la enseñanza de la disciplina (y no sobre la disciplina), vincular las prácticas pedagógicas de formación inicial con los conocimientos didácticos, impulsar ofertas de formación de posgrado en didácticas específicas y, tensionar al sistema, para que así se le dedique una mayor cantidad de fondos a los proyectos de innovación e investigación en didáctica.

Sin embargo, entre la docencia y los esfuerzos constantes por validarnos en la comunidad académica, nos damos cuenta que no estamos apartando tiempo suficiente para usar los conocimientos didácticos en mi- 
rar y resignificar nuestras propias prácticas. En muchas ocasiones estamos tan preocupados de que el futuro profesor se apropie de los conocimientos didácticos, que olvidamos el uso de la didáctica en nuestros procesos de enseñanza.

Por ello, no resulta sorprendente que los futuros profesores manifiesten que algunos cursos de didáctica son el contraejemplo de lo que no se debe hacer en una clase, en especial, porque se convierten en espacios de formación con lecturas que no son discutidas ni contextualizadas a la realidad de las escuelas; o en otros casos, a la estructuración de secuencias didácticas idealizadas que siguen las propuestas de diversos autores, pero que son inservibles en los contextos escolares (Jiménez-Tenorio \& Oliva, 2018).

Escuchar a los profesores en formación referirse a los cursos de didáctica y sus profesores, como antítesis de la didáctica, significó una preocupación que nos llevó a preguntarnos preliminarmente: ¿cuáles son los espacios reales que dedicamos a reflexionar sobre nuestra docencia universitaria?, ¿existe coherencia entre nuestro discurso sobre la didáctica y nuestras prácticas pedagógicas?, ¿cuáles son nuestros principales aciertos y dificultades en las aulas universitarias al momento de enseñar?, ¿cómo atendemos a las necesidades formativas de nuestros estudiantes?, ¿cómo desarrollan su trabajo profesional otros didactas de nuestra propia institución?, ¿qué podemos aprender de didactas de otras instituciones?

Como plantea Vanegas (2016), la reflexión sobre el conocimiento didáctico resulta clave, porque nos permite transformar las clases de matemática y ciencias y, además, nos ayuda a comprender las configuraciones identitarias. El profesor de matemática o de ciencia debe reconocer que el conocimiento propio en tanto profesional, no es la matemática o la ciencia (lo que es acertado para un matemático o un científico), sino el conocimiento didáctico del contenido que le permite hacer transformaciones adaptativas a los conocimientos disciplinares para que se puedan aprender en el contexto escolar o universitario.

Aunque los profesores compartimos muchos conocimientos (pedagógico, curricular, valores y principios de la educación, de los estudiantes, entre otros), el conocimiento didáctico del contenido es el que nos diferencia de otras profesiones y de otros profesores (Porlán, 2018; Sanmartí \& Marchán, 2015; Vergara \& Cofré, 2014). Sin embargo, este conocimiento es el que nos debería permitir reflexionar constantemente sobre nuestras propias prácticas, de tal manera que podamos innovar en nuestras aulas (Arana, Macías \& Martínez, 2018). 
A partir de lo anterior, hemos acordado que, para nuestro desarroIlo profesional docente y la mejora de nuestros roles como formadores de formadores, es prioritario responder a la siguiente pregunta de investigación: ¿Cuáles son los procesos reflexivos de didactas de las ciencias experimentales y matemática, cuando desarrollan un self-study colaborativo interinstitucional a partir de las actividades de docencia universitaria? Para responder a ello, nos hemos trazado como objetivo general, caracterizar los procesos reflexivos que vivenciamos didactas de las ciencias experimentales y matemática, cuando desarrollamos un self-study colaborativo interinstitucional a partir de las actividades de docencia universitaria.

\section{Contextos y Supuestos de los Participantes}

Dos años antes del inicio de esta investigación, el equipo de amigos críticos se había venido consolidando en instancias académicas, donde pudimos detectar que tenemos formas diferentes de analizar las situaciones de aula y la formación de profesores, y en medio de esa diversidad, generamos ambientes de confianza en los que la crítica argumentada y la reflexión nos han permitido analizar, repensar y comprender desde otros puntos de vista, nuestra labor como didactas. En estas interacciones, la riqueza del trabajo ha surgido debido a que cada uno de nosotros posee contextos particulares que le dan un posicionamiento diferente frente a los acontecimientos derivados de nuestra docencia y que ponen en tensión las prácticas o supuestos que parecen naturalmente cómodos (Vanegas y Fuentealba, 2018). En este escenario, se presentan los contextos particulares de cada didacta para que se comprendan los puntos de referencia desde donde emergen los datos, así como las vivencias que validan la interpretación y resignificación de la propia práctica, como puente de anclaje del trabajo colaborativo.

\subsection{Contexto y supuestos del investigador 1}

Soy Carlos, elegí como profesión ser profesor de matemáticas y física. Como profesor y como formador de profesores he nutrido dos corpus de conocimiento que me desafían: las didácticas de las ciencias experimentales y matemáticas y, la reflexión docente. La visión epistemológica kuhnniana de ciencia y didáctica, confirman mi primer supuesto sobre la necesidad de mejorar la enseñanza considerando factores personales y sociales de los profesores en formación y en ejercicio (Berry \& Van Driel, 2013; Mellado, 2003). La reflexión es una concepción que he construido teóricamente, que he vivido, que he dinamizado y observado en otros (Vanegas, 2016), por tanto, el segundo supuesto es que la reconozco como parte de mi identidad profesional 
El desarrollo de esta investigación interinstitucional mediante diseño de self-study, se convirtió en una oportunidad única para darle forma a mi propia reflexión pedagógica, y al mismo tiempo, me motivó trabajar con otros didactas ya que también están realizando diversos esfuerzos por impactar su propio desarrollo profesional y el de los futuros profesores de ciencias y matemática del país.

El contexto particular sobre el cual se realiza mi self-study corresponde a mi desempeño como formador en el curso de Cálculo III de una universidad pública de la Región Metropolitana de Santiago de Chile, durante el primer semestre de 2019. Los estudiantes del curso son futuros profesores de Matemática y Computación. Con algunos de ellos ya había tenido la oportunidad de tener la relación profesor-estudiante en la asignatura de Didáctica del Cálculo, por lo cual, este nuevo espacio de formación entrega un escenario diferente y genera el tercer supuesto de mi quehacer profesional: hay una tensión permanente para mí, puesto que, en el curso matemático de Cálculo III debo ser coherente con lo que enseñé en el curso de Didáctica del Cálculo.

\subsection{Contexto y supuestos de la investigadora 2}

Soy Carolina, profesora de matemáticas y doctora en didáctica de la matemática. En 10 años de experiencia en la formación inicial y continua de profesores de matemáticas, he realizado labores de docencia, investigación y algunas funciones en la gestión académica. He tenido la oportunidad de trabajar en didáctica de la matemática, vinculada a las prácticas pedagógicas, y a algunos cursos disciplinares. Desde el año 2018 trabajo en el centro sur del país, en una nueva escuela de Pedagogías, que desafía la construcción y organización de las carreras que la conforman.

El presente self-study se situó en un curso de Didáctica de la Matemática durante el primer semestre del 2019, en la carrera Pedagogía en Matemáticas. El objetivo de la unidad es el diseño y análisis de situaciones de referencia para la enseñanza y aprendizaje de la matemática de educación secundaria, centrado en el dominio del currículo de Educación Media: Estadística y Probabilidad. La unidad contempla el estudio de un marco teórico y metodológico que permite dar sustento y profundizar en los análisis que se realizarán, con énfasis en una habilidad del currículo escolar. Este marco se denomina Espacios de Trabajo Matemático (ETM) (Henríquez \& Montoya, 2015; Kuzniak, Tanguay \& Elia, 2016), el cual se utiliza en una perspectiva para el análisis del trabajo matemático del profesor y de los estudiantes. 
Mis supuestos de partida se relacionan, por una parte, con la relevancia de acercarnos y dar sentido a los contextos socioculturales locales en la formación de profesores y, al mismo tiempo, con la complejidad de instalar en el escenario nacional dicho campo de investigación con un sello propio.

\subsection{Contexto y supuestos de la investigadora 3}

Soy Carla, profesora de física y me he especializado en didáctica de la matemática y las ciencias experimentales. Participé de este self-study motivada por la oportunidad de analizar la propia práctica y su impacto en la formación científica; lo que significa como primer supuesto, la posibilidad de establecer y participar de un grupo de trabajo con espíritu crítico que, a través de reflexión conjunta basada en la experiencia, aporte a la mejora de la formación universitaria.

En el último tiempo mi trabajo se ha centrado en el impacto que tiene la implementación de estrategias de aprendizaje activo en el aprendizaje de la física. Esto me ha llevado a realizar procesos de investigación-acción junto a profesores de física en ejercicio a nivel escolar y universitario, logrando que reflexionen sobre sus propios paradigmas y estilos didácticos de clase como un motor para lograr transformaciones en la práctica.

El self-study ya presentado, estuvo enfocado en mi rol de profesora de física en el curso de Física General de una carrera de la Facultad Tecnológica. En este marco hay dos antecedentes importantes a considerar: Primero, que la asignatura se imparte para el estudiantado que haya reprobado el curso en el semestre anterior, es decir, que los inscritos son alumnos cuyo resultado académico en física no fue satisfactorio. Segundo, que por primera vez este curso fue impartido en las nuevas salas de Aprendizaje Centrado en el Estudiante (ACE) de la Universidad. Esto supone, y desde ahí lo que sustenta mi práctica, transformar la experiencia de los estudiantes de clases tradicionales, a un nuevo espacio y forma de aprender, donde pasan de ser sujetos pasivos a sujetos activos y protagonistas de las clases.

\subsection{Contexto y supuestos de la investigadora 4}

Soy Carolina, profesora de biología. Desde hace aproximadamente doce años, trabajo e investigo en el contexto de la educación superior, vinculada a la formación inicial de profesores de ciencias. Actualmente, soy docente en una universidad pública de la zona centro sur de Chile. El inicio de mi trabajo en la universidad no ha estado exento de tensiones y desafíos, mis experiencias, las que me han hecho transitar en un cambio permanente para resignificar el sentido de la propia práctica pedagógica e investigativa. 
En tal sentido, uno de los supuestos iniciales sobre los que baso mi actuar como formadora, se relaciona con pasar desde la visión de la educación en ciencias como práctica, la que se condice más con la idea de un hacer y cuyo actuar se puede volver instrumental, a una visión centrada en la experiencia, la que supone para mi alegría, dar espacio para el pensamiento, para una vivencia que nos pasa, que nos sucede, que nos pone en crisis; y que nos abre la posibilidad de escuchar aquello que verdaderamente nos importa y nos invita a reflexionar y a transformarnos (Contreras y Pérez de Lara, 2010).

Mi vivencia como docente formadora de futuros profesores/as de ciencias experimentales se constituye como el escenario en el cual se lleva a cabo el presente self-study colaborativo, poniendo en el centro del análisis a mis propias prácticas docentes y experiencias en la asignatura vinculada a la temática de identidad profesional. En este proceso y desde la perspectiva de interactuar junto a otros en la mirada reflexiva de la propia práctica, la relación con los amigos críticos y la construcción de una amistad crítica ha sido vital en el aprendizaje conjunto de nuevas experiencias profesionales (Shuck y Russell, 2016), abriéndonos a la posibilidad de volver a descubrir nuevos significados y formas de actuación.

\section{Metodología}

La investigación se realizó desde un paradigma cualitativo con enfoque de self-study (Olarte, 2016; Russell, Fuentealba \& Hirmas, 2016; Russell \& Mena, 2016). Se trata de interpretar críticamente el papel de nosotros como formadores de formadores, analizando las consecuencias de la toma de decisiones personales, así como las características y matices de los contextos particulares en los que se desarrolla nuestra docencia universitaria.

El diseño metodológico de la investigación se ha estructurado en dos fases. Como mostramos en la figura 1, hemos adaptado el ciclo deweyniano (Vanegas y Fuentealba, 2018) de tal manera que el proceso de reflexión pasara primero por la mirada personal, y posteriormente, las nuevas comprensiones emergidas del primer ciclo reflexivo, fueran sometidas a prueba mediante un ciclo de reflexión colaborativa con los amigos críticos, lo cual, permitió resignificar las nuevas comprensiones y determinar efectos en supuestos y acciones de la propia práctica y la de los amigos críticos. A continuación, se detallan los instrumentos y procedimientos que nos permitieron Ilevar a cabo cada fase del estudio. 


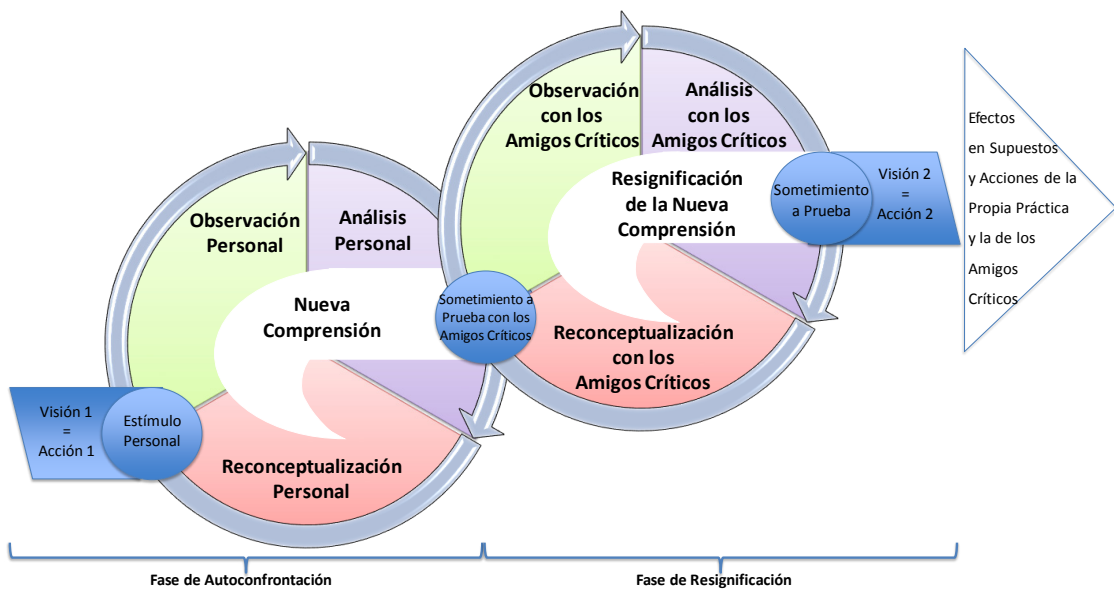

Figura 1. Ciclo Reflexivo para un Self-Study Colaborativo. Fuente: elaboración propia a partir del modelo deweyniano (Vanegas y Fuentealba, 2018, p. 17).

Fase I: Autoconfrontación. Para identificar aciertos, dilemas, contradicciones y tensiones que emergen durante nuestra docencia universitaria, durante un mes, cada uno de los didactas del proyecto realizó una bitácora sobre el desarrollo de sus clases. Para ello, luego de finalizar las actividades de clase, respondimos ante una grabadora de voz las siguientes preguntas:

- ¿Qué situaciones me llamaron la atención?

- ¿Qué sentimientos o emociones me generó cada situación?

- ¿Por qué es importante que registre este evento?

- Si tuviese que enfrentarme de nuevo a una situación similar, ¿la enfrentaría de la misma manera?

- ¿Considero que la clase fue coherente con la didáctica de mi disciplina?

- A partir de las decisiones planteadas en registros anteriores, ¿cuáles cambios logré implementar? ¿cómo resultaron y por qué?

Complementariamente, en cada clase el didacta solicitó a 5 estudiantes (seleccionados al azar) que completaran un 'ticket de salida' en el que se les preguntó cómo les pareció la clase, qué aprendieron, qué preguntas les surgen a partir de la clase, cómo se sintieron y qué recomendarían al didacta para mejorar su docencia.

Fase II: Resignificación. Una vez finalizado el mes de la toma de datos, los registros de audio fueron transcritos y procesados por cada didacta a través del análisis del contenido del discurso (Menéndez \& Rodríguez, 2012; Santander, 2011). Para ello, usamos 3 categorías y 4 subcategorías emergentes (Anexo 1). 
La sistematización de este análisis fue presentada por cada didacta ante el equipo de amigos críticos en una jornada de reflexión colaborativa que sirvió, desde el punto de vista metodológico, para triangular la información (Vallejo y Finol de Franco, 2009), y desde el punto de vista reflexivo, para resignificar colaborativamente las nuevas comprensiones, a través de la observación, el análisis y la reconceptualización con los amigos críticos (Hirmas \& Blanco, 2016; Russell, 2018; Vanegas y Fuentealba, 2018). La jornada de reflexión colaborativa fue videograbada y, similar a la fase 1 , analizada a través del análisis del contenido del discurso (Santander, 2011; Menéndez \& Rodríguez, 2012), de tal manera que se pudiesen caracterizar las resignificaciones que vivió y reconoció cada didacta.

\section{Resultados y análisis}

Para respetar las particularidades de los procesos reflexivos de cada didacta, así como evitar las generalizaciones reduccionistas que violentan los self-study (Olarte, 2016; Russell, Fuentealba \& Hirmas, 2016), presentaremos en cada caso lo que ocurrió en la fase de autoconfrontación y en la fase de resignificación. Iniciaremos con didactas de la matemática y finalizaremos con didactas de las ciencias experimentales.

\subsection{Autoconfrontación y resignificación del investigador 1}

El primer ciclo reflexivo me permitió reconocer que las tensiones y transformaciones de mi docencia universitaria están fuertemente marcadas por las subcategorías relacionadas con los estudiantes y la didáctica específica. En los tickets de salida me vi sorprendido por demandas de los estudiantes que creí que las tenía resueltas o me parecían irrelevantes, tales como: generar mayores apoyos visuales para los conceptos abstractos, plantear ejemplos que fuesen coherente con las pruebas de las evaluaciones escritas, plantear sólo ejercicios con alto nivel de dificultad, darles tiempo para tomar notas de las proyecciones o de lo que escribía en la pizarra y, no dar libertad de elegir los problemas que se resolvían en algunas clases.

Algunas de las anteriores demandas Ilevaron a resignificaciones y adaptaciones puntuales de los supuestos 1 y 3 . Los dos primeros aspectos me preocuparon mucho, porque contradecían dos aspectos de mi epistemología personal como didacta de la matemática: primero, la teoría de los registros de representación semiótica (Duval, 2006), en la cual me baso para generar distintos modos de crear modelos mentales, representacionales e interpretativos de los conceptos matemáticos; segundo, el triángulo didáctico 
entre la enseñanza, el aprendizaje y la evaluación (Porlán, 2018; Sanmartí \& Marchán, 2015), sobre el cual siempre estoy alerta para ver la coherencia de mi docencia y la pertinencia de los procesos de transposición didáctica (Chevallard, 1999). Para la demanda de generar mayores apoyos visuales, consideré que, aunque lo hacía, era necesario generar más y de distinto tipo, es decir, esta tensión me llevó a intencionar un cambio específico durante el curso. En cuanto a la recomendación sobre los ejemplos coherentes con las evaluaciones, no estuve de acuerdo en realizar cambios, porque no encontré situaciones que evidenciaran alguna incoherencia. Sin embargo, como plantearé más adelante, la visión de mis amigas críticas me ayudó a entender las razones por las cuales apareció ese comentario.

El comentario que más me ayudó a resignificar mis supuestos fue el del nivel de dificultad de los ejercicios que resolvía en clase. Este comentario fue recurrente, no obstante, al ahondar en lo que pretendían decir los estudiantes, en realidad me mostraron que tengo muy marcado el abordaje de contenidos desde lo simple a lo complejo y desde lo concreto a lo abstracto, lo cual lo atribuyo a la apropiación que tengo del ciclo de aprendizaje constructivista de Jorba y Sanmartí (Sanmartí \& Marchán, 2015). Por tanto, la demanda de los estudiantes era hacia la innovación didáctica mediante procesos de enseñanza en el que invirtiera el ciclo: desde lo complejo a lo simple y desde lo abstracto a lo concreto.

Los tickets fueron de alto valor para este primer ciclo reflexivo, porque también me permitieron confrontar mis procesos afectivos a la luz de los procesos afectivos de los estudiantes. En coherencia con mi primer supuesto, la voz de los estudiantes y las dimensiones personales (de ellos y la mía) marcan mi profesionalidad como docente y la configuración identitaria como formador (Rusell, 2018; Vanegas \& Fuentealba, 2019).

El análisis de las clases y de los tickets me llevaron a concluir que, generalmente, había coherencia entre mi acción docente y la didáctica de la matemática. Sin embargo, al estar analizando los datos, me llamó mucho la atención el valor que le doy al contrato didáctico (Ávila, 2001). Este es un concepto central que trabajo en los cursos de Didáctica de la matemática o de las ciencias, el cual defino como el conjunto de convergencias y divergencias, implícitas y explícitas, que se generan en la interacción de los sistemas didácticos (profesor, estudiantes y saber). Por tanto, mi tercer supuesto, más que pensarlo como una tensión entre el cálculo y su didáctica, ahora lo veo como parte de la vigilancia epistemológica sobre el contrato didáctico.

El segundo ciclo reflexivo fue un proceso de reflexión en voz alta que, al ser analizado desde la perspectiva de mis amigas críticas, me permitieron alcanzar resignificaciones de mayor relevancia para mi desarrollo 
profesional. Gracias al trabajo colaborativo pasé de pensar en la preocupación por la coherencia con la didáctica de la disciplina (supuesto 3) a situarme desde el conocimiento especializado del profesor de matemática (MTSK por sus siglas en inglés) como corpus de saber más complejo (Muñoz, Contreras, Carrillo, Rojas, Montes \& Climent, 2015).

Otro aspecto que logramos resignificar fue la forma como estaba abordando las contradicciones o tensiones con los estudiantes, en particular aquellas en las que no podía estar de acuerdo, lo que fue explicado por una de mis amigas críticas de la siguiente manera: "al final tú le viniste a generar a ellos un quiebre de paradigma de lo que son las asignaturas disciplinares" (Amiga crítica 1).

Cuando les planteé a mis amigas críticas que me tenía conflictuado el hecho de que los estudiantes me pidieran aumentar el nivel de dificultad de los ejercicios que resolvía en clase, sus puntos de vista me ayudaron a ver la situación desde otra perspectiva. De nuevo, mi epistemología personal sobre el rol de la didáctica en la formación de profesores de matemática, me lleva a comprender los grados de dificultad del aprendizaje matemático desde una concepción muy diferente a la de los estudiantes, ya que su punto de referencia para hablar de dificultad está puesta en las experiencias con otros cursos disciplinares donde el acento no está en la construcción del conocimiento, sino en el abordaje demostrativo de teoremas y ejercicios complejos que se debían reproducir en una prueba escrita.

Con respecto al tercer supuesto, en el siguiente relato hay un Ilamado de atención de mis amigas críticas a ser cauteloso con las formas de ver los obstáculos o no cumplimiento de expectativas durante la docencia universitaria.

Carlos: yo decía, ahí me faltó algo de mi parte.

Amiga crítica 1: para ser justo con lo que estabas haciendo, estabas buscando estrategias que no existen o que no es lo usual, y eso es normal cuando lo miras desde la didáctica.

Amiga crítica 2: te falta darte el tiempo de hacer el curso de nuevo, o sea, no seas tan ambicioso de pensar que lo tienes que resolver.

Este espacio de discusión con las amigas críticas fue de mucho alivio para mí, puesto que me ayudaron a entender que poseer algunos conocimientos de didáctica de la matemática no es sinónimo de tener resuelto o saber responder a todos los desafíos implicados en la enseñanza y el aprendizaje de un objeto matemático. Cuando miro los tres supuestos con los que partí el estudio, veo que el trabajo colaborativo con las amigas críticas logró llevarme a un plano de desafío profesional en el que están implicados mi 
epistemología personal, el desarrollo del MTSK, la investigación didáctica y mis construcciones identitarias con formador de formadores (Hirmas \& Blanco, 2016; Russell, 2018).

\subsection{Autoconfrontación y Resignificación de la investigadora 2}

Para los análisis de contradicciones y tensiones en el primer ciclo reflexivo, mi foco está en dos clases específicamente, ubicadas al inicio del estudio de los ETM, empleado como una herramienta teórica y metodológica para el análisis del trabajo matemático. En específico, en la primera sesión de dicha unidad, los estudiantes trabajaron de manera colaborativa en la resolución de problemas matemáticos y luego, presenté de manera expositiva algunos elementos teóricos de los ETM, para analizar el trabajo de sus producciones. En la segunda sesión me enfoqué en mostrar algunos ejemplos sustentados en el ETM (Kuzniak, et al., 2016), y en los alcances del trabajo teórico-práctico que los estudiantes realizarán, el cual involucra el diseño, experimentación y análisis de una propuesta de situaciones de referencia para la enseñanza.

Para estos análisis me focalizo en la subcategoría de la didáctica de la matemática, vista como una disciplina científica (Bielher, Scholz, Strässer \& Winkelmann, 2002); es decir, que estudia problemáticas de la enseñanza y aprendizaje desde distintos niveles y perspectivas (Hodgson, Kuzniak \& Lagrange, 2016), y cuyo foco está en el estudio de problemáticas ligadas a la matemática (su enseñanza y aprendizaje).

En particular, los estudiantes consultaron y se interesaron con el tema teórico (ETM), sobre todo, por tratarse de un trabajo que conecta con la Evaluación Nacional Docente. No obstante, aquí identifico una paradoja entre mi visión y la opinión que recojo de algunos estudiantes, pues yo usualmente muestro ejemplos o aplicaciones de la componente teórica en juego ${ }^{1}$, pero no de todo, ni de cada tema o concepto que se estudia en las clases. Los estudiantes me han manifestado que desean que muestre todo, con ejemplos concretos y definiciones. Yo presento/explico/cito trabajos de otros investigadores que han realizado aportes relacionados con el tema, describo qué hacen y cómo se relaciona con el tema que estamos estudiando; mi intención es conectar la disciplina, pero al mismo tiempo, motivar la indagación y búsqueda de trabajos o investigaciones relacionadas con el tema.

${ }^{1}$ Cuando hablo de componente teórica me refiero a una teoría o una línea de investigación de la didáctica de la matemática, en un sentido amplio. 
En los tickets de salida los estudiantes destacan aspectos teóricos aprendidos, y cuando se preguntan cómo se sienten en la clase, en general señalan que se sienten cómodos, dicen: "bien", "cómodo", "incluida", "motivado al inicio". También escriben que: "aprenden los temas", "se sienten interesados", "son parte de la clase". Esto me genera satisfacción por el trabajo que he realizado en estas clases.

Finalmente, mi postura epistémica (como didacta de la matemática), que podría llamar como "mi postura invariante", creo (y lo he constatado a lo largo de los años) que los estudiantes pueden generar, crear, innovar, o desarrollar investigaciones pedagógicas de las prácticas, basados en sus propios ejemplos, tareas y errores. De acuerdo con mi experiencia, esto me ha dado favorables resultados, los estudiantes (de formación inicial y continua) logran desarrollar sus propias aproximaciones, que hacen sentido desde su propio contexto, sin la necesidad de mostrar modelos para cada temática de estudio. Esta dinámica de trabajo, requiere reflexión y una revisión más exhaustiva y profunda de mi parte, pero los resultados me confirman que es una buena oportunidad para generar conocimiento.

En cuanto al segundo ciclo reflexivo, después de analizar los focos de contradicciones y tensiones antes descritos, presento cinco puntos que han sido analizados en conjunto con mis amigos críticos y también, lo planteo como desafíos a trabajar:

1. Mi postura epistémica como didacta de la matemática y el rol en la formación del profesorado de la disciplina.

2. Considerar dentro de mi planificación y trabajo formativo con los estudiantes, mi propia investigación.

3. Involucrar de manera explícita y efectiva los contextos socioculturales locales de los estudiantes en formación inicial.

4. Considerar como parte de mi organización de la enseñanza, aspectos en los que inciden los contextos locales, tales como: las necesidades académicas, sociales y familiares de los estudiantes y, su incidencia en la formación del profesorado.

5. Involucrar a los estudiantes en más actividades académicas con estudiantes de otras instituciones.

De estos resultados, los objetivos planteados en este self-study, y mis propios supuestos de partida, destaco los aspectos que han permitido considerar elementos nuevos, o replantearme la manera en que éstos estaban siendo considerados en mi quehacer y, otros en los que mi experiencia en la formación inicial y continua del profesorado de matemáticas me han llevado a tomar una postura en mi rol de didacta de la matemática. 
En cuanto a los dos primeros desafíos, me parece pertinente destacar cómo a través de esta experiencia, he podido robustecer mi postura como formadora de profesorado de matemáticas en el dominio de la didáctica de las matemáticas y he decidido trabajar con mi propia línea de investigación, pues creo importante que los estudiantes aborden la disciplina a partir de la propia experiencia de sus formadores.

En particular, ha sido un trabajo constante el modo en el que logro articular aspectos teóricos con la práctica del profesor para la formación inicial, y poder emplearlos como herramientas teóricas que permitan al futuro profesor la toma de decisiones sustentadas en conocimiento científico.

En cuanto a "mi postura invariante", mis amigos críticos me han llevado a reflexionar sobre la importancia de mantenerla, considerar mi experiencia y conocimiento y, a partir de ello, poder explicitarla sin el peso negativo que tuvo en algún momento para mí. Esto tiene relación con favorecer el desarrollo de competencias basadas en investigación de la propia práctica de un profesor y su conocimiento especializado, que le permitan tomar decisiones argumentadas desde distintas perspectivas, reflexionar y analizar casos, basados en información proveniente de contextos reales.

Finalmente, destaco las reflexiones durante y post movilizaciones estudiantiles, las que han incidido para analizar con foco en la didáctica de la matemática y contextos locales de los estudiantes, pues me pareció inconcebible en dichos fenómenos que emergen de la transposición didáctica (Chevallard, 1999), los contextos escolares sin alumnos o sin profesores o sin escenarios, pero además, dichos escenarios o contextos deben ser analizados con profundidad en su dimensión local; no abordarlo de esta manera sería un sinsentido para mí. Este punto es particularmente relevante a mi juicio, y queda aún trabajo por hacer; sin embargo, creo que contribuye en esa dirección el dar sentido a la propia práctica a partir de ejemplos, investigación y escenarios locales.

\subsection{Autoconfrontación y Resignificación de la investigadora 3}

Para mi primer ciclo reflexivo, es muy importante considerar el contexto y los supuestos antes planteados, para comprender las posibles tensiones que surgen con respecto a las clases ya que la docencia se realizó con metodologías de aprendizaje activo. Esto implicó trabajar con el modelo de Aula Invertida que supone resistencias y dificultades para los estudiantes, sobre todo al inicio del curso (Hernández y Tecpan, 2017). 
Primero, en un curso activo no se presentan/exponen contenidos durante la clase, sino que los contenidos están disponibles para consulta a través de apuntes y videos que yo les comparto en una plataforma virtual y que pueden consultar previamente. De este modo, la clase sólo está enfocada en el trabajo mayormente colaborativo de parte del estudiantado con actividades especialmente diseñadas en función de los aprendizajes que se van obteniendo. En ese sentido, me he dado cuenta que mis clases son coherentes con un modelo didáctico de aula invertida, basado en un paradigma socioconstructivista, que coincide con mi segundo supuesto de estudio.

Frente a este contexto, surgen dos reflexiones importantes en el primer ciclo de análisis. Por una parte, la diferencia que conlleva enseñar una disciplina en particular (física en este caso) cuando es pensada desde la didáctica específica. Esto implica tener en cuenta consideraciones metodológicas, modelos y creencias del estudiantado respecto a cada contenido que se espera enseñar. Por otra parte, la reflexión respecto a lo que implica centrar las clases en el aprendizaje del estudiante.

Considerando que los sentimientos y emociones emanadas de los tickets de salida fueron favorables y reflejan una percepción positiva de los estudiantes respecto a las clases, es interesante destacar las respuestas que surgen a una segunda pregunta relacionada con recomendaciones que ellos me harían. Las clases activas siempre genera en los estudiantes visiones dicotómicas, es decir, hay algunos a quienes les gusta ser los actores y protagonistas de la clase y otros a los que les cuesta acostumbrarse, especialmente porque demanda mayor interacción y autorregulación. En este sentido, los tickets aplicados confirman esta dicotomía y a la vez, ratifican mi segundo supuesto de estudio, ya que la mayoría de los comentarios de los estudiantes son positivos respecto a las clases, salvo uno que preferiría las clases tradicionales.

La percepción que los estudiantes tienen respecto a lo que es aprender física en contextos de aula invertida, y lo que implica eso para mí como docente de la asignatura, genera una tensión que conlleva un proceso de reconocimiento y cuestionamiento desde la didáctica específica, considerando la coherencia que mis clases tienen con principios, modelos, teoría y la epistemología desde las cuales las planifico. Por ejemplo, la selección de actividades a realizar en la clase se realiza sobre la base de la literatura especializada que ha identificado cuáles son las principales dificultades de aprendizaje que tienen los estudiantes cuando se enfrentan a ciertos temas disciplinares particulares, por ejemplo, equilibrio estático, fluidos, u otros. 
A partir del análisis anterior, hay dos ideas potentes que surgen durante mi segundo ciclo reflexivo, que determinan la reflexión sobre la didáctica como cuerpo de conocimiento. En la jornada de reflexión colaborativa planteé a mis amigos críticos una situación que he categorizado como 'sentimiento', pero que se relaciona con la subcategoría definida como didáctica específica:

Me llamó la atención la disposición que tenían los estudiantes para poder terminar el semestre a tiempo, ya que el resultado de la prueba 2 no fue buena, pues reconocieron que no se habían preparado lo suficiente porque habían tenido otras pruebas esa semana, entonces estaban resignados a que la prueba 2 no iba a ser buena, pero con mayor razón venían a la clase interesados en aprender lo necesario para aprobar luego la prueba 3 y mejorar sus resultados

Por una parte, esta experiencia denota una emoción para mí como didacta porque genera sorpresa la determinación de los estudiantes para continuar con el semestre y no abandonar la asignatura frente a un mal resultado de su segunda evaluación. Por otra parte, representa un desafío desde el punto de vista de la preparación de las clases siguientes, que tensiona las decisiones metodológicas desde la didáctica específica. En este sentido, los amigos críticos señalan ideas importantes que invitan a la reflexión:

Amiga crítica 1: la didáctica es un cuerpo de conocimiento que da sustento a las decisiones pedagógicas, acciones y tareas que se desarrollan en clases. Existe un rol del didacta que permea tus clases

Amigo crítico 2: la didáctica como disciplina no es sólo un campo de investigación. Si yo investigo para nutrir ese campo no puedo hacer clases con acciones que contradigan la teoría que sustenta la investigación y su epistemología

Entonces, Ilegamos a la conclusión de que la formación en didáctica nos lleva a reflexionar, analizar y transformar constantemente la práctica para mejorar la calidad de los aprendizajes. Esto se visualiza al considerar que las clases realizadas por un didacta se piensan y planifican en coherencia con modelos y paradigmas educativos propios de la didáctica específica.

La didáctica influye en la clase no sólo desde las decisiones metodológicas, sino que también como cuerpo de conocimiento y campo de investigación que determina el actuar del docente y nutre la práctica pedagógica. Frente a la discusión, logramos determinar como equipo de amigos críticos, que la práctica pedagógica de un didacta conlleva una epistemología que se actualiza constantemente y que es tensionada por la didáctica específica, independientemente de que la clase sea para formación docente, de ingeniería, o cualquier otro contexto. Este resultado confirma mi primer supuesto, respecto a lo esencial de contar con espacios de discusión como el realizado en el ciclo II de este self-study. 


\subsection{Autoconfrontación y resignificación de la investigadora 4}

En común acuerdo con Berry (2016), las tensiones reconocidas e identificadas durante mi primer ciclo reflexivo emanan al momento de intentar conciliar los objetivos de la enseñanza de los futuros docentes, con las necesidades y expectativas que ellos mismos tienen y expresan sobre su formación. En este escenario sumo, además, una tensión relacionada con el impartir docencia a estudiantes en estadios iniciales de la carrera de pedagogías en ciencias, lo que me lleva a asumir prácticas que enfatizan en la relación pedagógica y el sentido de la profesión. Otra cuestión importante es poner atención sobre la escucha a quienes están aprendiendo a enseñar (Russell, 2018).

El curso tenía una planificación que a mi juicio era apropiada, sugería actividades y lecturas pertinentes. Yo buscaría generar algunas experiencias en donde los estudiantes pudiesen visualizar la identidad de otros docentes de ciencias del sistema escolar, y que esta interacción nos diera insumos para discutir y reflexionar en nuestras clases. Durante la primera semana decidí aplicarles una encuesta que recogía información acerca de su contexto sociocultural y de sus motivaciones por estudiar pedagogía. Al leer y revisar los resultados de la encuesta, aparece la primera tensión. Mis estudiantes me comentaban que la pedagogía en ciencias no era su primera opción profesional, sino más bien, algo que estaban probando, evaluando y viendo si les gustaba.

Estudiante 1: la verdad es que pedagogía es mi segundo intento de carrera, pero no por ello, menos importante

Estudiante 3: No sé si estoy tan seguro de terminar la carrera y ejercer la profesión...

Estas respuestas me hicieron cuestionar la planificación de la asignatura, y asumir el desafío de cambiarla. Intento entonces responderme, siguiendo algunas prácticas de otros docentes (Bain, 2005): ¿Qué capacidades y habilidades desarrollarán mis estudiantes en estas clases?, ¿Cómo podré incentivar su interés? ¿Qué preguntas importantes les ayudará esta docencia a responder?

Cambio la planificación con la intención de desafiar los estereotipos y expectativas de la profesión docente:

1. Incluir lecturas asociadas al sentido de la vocación pedagógica. Trabajé con el libro de Paulo Freire "Cartas a quien pretende enseñar".

2. Decidimos entrevistar a un profesor de especialidad que realice clases en la escuela. Construimos conjuntamente pauta de 
entrevista con foco centrado en rasgos de la construcción de la identidad profesional atendiendo a sus propias situaciones de vida cotidiana, sus discursos, sus sensaciones, percepciones y modos de ser y estar en el espacio profesional (Veiravé, Ojeda, Nuñez y Delgado, 2006).

3. Les desafié a llevar una bitácora de la clase, a la cual llamamos bitácora narrativa. Como no conocían esta estrategia, debimos conciliar una estructura guía, que les permitiera reflexionar acerca de sus experiencias en la clase, y al mismo tiempo, acerca de esta construcción de identidad. La idea era relacionar sus historias de estudiantes y profesores en formación, con sus historias personales (Cornejo, 2016). Dimos espacio en clases, para conversar acerca de lo que iban registrando, y a menudo, yo intentaba desafiarles con algunas preguntas y retroalimentar de forma escrita sus narraciones (Bain, 2005).

4. Acordamos que harían una visita a la Escuela, e intentarían familiarizarse con el entorno y con la profesión del profesor de ciencias. En ese contexto, construimos conjuntamente una pauta de observación abierta, más bien orientada para realizar un registro etnográfico (Jackson, 1998).

5. Incorporé como parte de la secuencia de la clase, la aplicación de los tickets de salida de los estudiantes.

Cuando comienzo a recoger información acerca de las clases, y a mirar las producciones de los estudiantes, aparecen nuevas tensiones acerca de la dedicación que realizan a las asignaturas de especialidad científica. Reconocen un amor profundo por las ciencias, pero no necesariamente por su enseñanza. Dado este escenario, vuelvo a implementar otros cambios. Así que, elaboro un trabajo de cierre de la asignatura, desde la terapia narrativa, mediante la estructuración de un árbol de la vida (Madrid, 2015). Este trabajo implicaba utilizar la metáfora del árbol, para narrar su propia historia vinculada a la educación, en tres escalas: una relacionada con sus raíces, su historia familiar y personal; otra vinculada al tronco, que los insta a identificar sus habilidades, talentos y experiencias significativas de su estar en la escuela; y finalmente las ramas, las que representan esperanzas y deseos vinculadas al docente que desean ser.

Al revisar las producciones de mis estudiantes, aparecen varios hechos significativos vinculados a su historia personal, 'su experiencia'; la que es valiosa en el marco de la construcción de la propia identidad profesional. Su narrativa expresa el reconocimiento de la labor docente, y cómo han vivido la experiencia de contar con diversos profesores que han "dejado huellas". 
Esto me hace reflexionar nuevamente acerca de cómo dar espacio a los futuros profesores durante su proceso formativo, tal como lo plantea Berry (2016), para crear conocimiento en el aprendizaje sobre la enseñanza, tomando como anclaje la experiencia. Esto me supone re-planear la asignatura en un futuro, poniendo atención a estos elementos, lo que me lleva a repensar los supuestos con los que inicio el estudio. Necesito generar conocimiento para acompañar a mis estudiantes a construir su incipiente identidad profesional como profesores de ciencias.

Las reuniones sostenidas con los amigos críticos durante mi segundo ciclo reflexivo, viene a retroalimentar lo que ya comentaba anteriormente. Me doy cuenta de que los supuestos iniciales con los que comienzo la asignatura, no se condicen con lo que realmente viven y experimentan mis estudiantes. Mis amigos me hacen ver que si bien yo cambio la planificación de la asignatura, en función de las necesidades de mis estudiantes, estos cambios más bien se dan en función de que los supuestos que yo tenía al iniciar el curso, no se pudieron cumplir. Yo creía que mis estudiantes podrían comenzar con una construcción identitaria de docentes, cuando aún tienen una identidad como estudiantes. Olvidé que la construcción de la identidad se inicia en la formación inicial y se prolonga durante todo el ejercicio de la profesión (Vanegas y Fuentealba, 2019). Tal como me lo expresa uno de mis amigos críticos "la identidad docente no se construye en un semestre de clases".

\section{Conclusiones}

El estudio nos ha permitido rescatar 'la voz en primera persona' de las experiencias que hemos vivenciado durante la investigación, y al mismo tiempo, dar cuenta de las diferentes maneras como enfrentamos, tensionamos y resolvemos desafíos que supone la docencia universitaria. De manera complementaria, en cada relato se evidencia el valor dado al trabajo colaborativo, en el cual, 'la voz de los amigos críticos' se convierte en el mejor aliado para darle profundidad, nuevos sentidos y significados a los procesos reflexivos sobre nuestras prácticas pedagógicas.

Como muestra la tabla $1 \mathrm{y}$, en coherencia con algunos autores (Hirmas \& Blanco, 2016; Russell, 2018; Vanegas y Fuentealba, 2018), la apuesta metodológica de adaptar el ciclo deweyniano en dos fases reflexivas, nos permitió explicitar los supuestos particulares y reconocer que la reflexión de cada didacta fue vivenciada de manera diferente durante la autoconfrontación, y al mismo tiempo, la reflexión con los amigos críticos durante la resignificación, hizo que aparecieran nuevas dimensiones reflexivas que llevaron a identificar supuestos comunes y cuestionar los supuestos particulares. 
En nuestro caso, el self-study colaborativo ha puesto de nuevo en tensión los tiempos que dedicamos a reflexionar de manera individual y colectiva sobre nuestras propias prácticas. Articular los tiempos de los cuatro didactas ha sido uno de los desafíos más complejos de este estudio. Incluso, el proceso de escritura de este artículo ha significado cuatro experiencias de aprendizajes diferentes que pasaron por los siguientes hitos: la búsqueda personal del sentimiento de contigüidad con los marcos teóricos y metodológicos, pensar y escribir en primera persona en un trabajo que es colectivo, saber recuperar la voz de los cuatro, mantener la preocupación por la coherencia y alineación del equipo, interpelar al otro en un marco de respeto y profesionalismo que le ofrezca la confianza, el deseo y los nuevos insumos para replantear o profundizar sus procesos de reflexión.

Estamos convencidos que el self-study colaborativo es una forma de desarrollo profesional que amplía perspectivas y nos compromete a los formadores con los propios valores y supuestos personales, así como con los del equipo de amigos críticos, lo que sustenta la construcción de nuevos roles y significados para nuestro trabajo académico como formadores de formadores.

\section{Agradecimientos}

A la Organización de Estados Iberoamericanos (OEI) por el financiamiento del proyecto y el acompañamiento durante su implementación.

\section{Referencias bibliográficas}

Arana, R. M., Macías, J. M. \& Martínez, K. M. (2017). Hacia una concepción didáctica reflexiva en la Educación Superior. Una experiencia desde el Ecuador. Opuntia Brava, 9(3), 147-154.

Ávila, A. (2001). El maestro y el contrato en la teoría brousseauniana. Educación matemática, 13(3), 5-21.

Bain, K. (2005). Lo que hacen los mejores profesores universitarios. Valencia: PUV.

Berry, A. (2016). Tensiones en la reconceptualización del conocimiento del formador de formadores: explorando la tensión entre valorar y reconstruir la experiencia. En: Russell, T., Fuentealba, R. \& Hirmas, C (Eds). Formadores de Formadores, descubriendo la propia voz a través del self-study. Santiago de Chile: OEI

Berry, A. \& Van Driel, J. H. (2013). Teaching About Teaching Science Aims, Strategies, and Backgrounds of Science Teacher Educators. Journal of Teacher Education, 0022487112466266. 
Bielher, R., Scholz, R., Strässer, R. \& Winkelmann, B. (2002). Didactics of Mathematics as a scientific discipline. New York: Kluwer Academic.

Contreras, J. y Pérez de Lara, N. (2010). La experiencia y la investigación educativa. En: J. Contreras y N. Pérez de Lara (Eds). Investigar la experiencia educativa. Madrid: Morata.

Cornejo, J. (2016). El Self-Study de la práctica de los formadores de futuros profesores: bases teóricas, características y modalidades metodológicas. En: T. Russell, R. Fuentealba, \& C. Hirmas (Eds). Formadores de Formadores, descubriendo la propia voz a través del self-study. Santiago de Chile: OEI

Chevallard, Y. (1999). El análisis de las prácticas docentes en la teoría antropológica de lo didáctico. Recherches en Didactique des Mathématiques, 19(2), 221-266.

Duval, R. (2006). Un tema crucial en la educación matemática: La habilidad para cambiar el registro de representación. La Gaceta de la Real Sociedad Matemática Española, 9(1), 143-168.

Guzmán, J. (2011). La calidad de la enseñanza en educación superior. Perfiles educativos, 33, 129-141.

Henríquez, C. \& Montoya, E. (2015). Espacios de trabajo geométrico sintético y analítico de profesores y su práctica en el aula. Enseñanza de las Ciencias, 33(2), 51-70.

Hernández, C. \& Tecpan, S. (2017). Flipped classroom mediated by the use of virtual platforms: a case study of pre-service teacher education in physics. Estudios pedagógicos (Valdivia), 43(3), 193-204. https://dx.doi.org/10.4067/S0718-07052017000300011

Hirmas, C. \& Blanco, R. (2016). Introducción. In J. Cornejo, J. Loughran, T. Russell, A. Berry, S. Sandretto, S. Schuck, . . J. J. Mena, Formadores de Formadores, Descubriendo la Propia Voz a través del Self-Study (pp. 7-11). Santiago: BUK Grafica \& Editora.

Hodgson, B., Kuzniak, A. \& Lagrange, J. B. (2016). The Didactics of Mathematics: Approaches and Issues. New York: Springer.

Jackson, P.W. (1998). La vida en las aulas. Madrid: Morata.

Jiménez-Tenorio, N. y Oliva, J. (2016). Análisis reflexivo de profesores de ciencias de secundaria en formación inicial en torno a diferentes secuencias didácticas. Revista Eureka sobre Enseñanza y Divulgación de las Ciencias, 13 (2), 423-439.

Kuzniak, A. Tanguay, D. \& Elia, I. (2016). Mathematical Working Spaces in schooling: an introduction. ZDM Mathematics Education, 48, 721-737.

Madrid, J. (2015). Construcción de la identidad narrativa usando el recurso del "Árbol de la vida", en estudiantes de la institución educativa "San Columbano", Lima, 2014. Revista de Investigación PAIDEIA en Ciencias Humanas y Educación, 1(1), 33-46.

Mellado, V. (2003). Cambio didáctico del profesorado de ciencias experimentales y filosofía de la ciencia. Enseñanza de las Ciencias, 21(3), 343-358.

Menéndez, M. A. \& Rodríguez, I. S. (2012). Metodología de la investigación social: técnicas innovadoras y sus aplicaciones: Síntesis.

Muñoz, M. C., Contreras, L. C., Carrillo, J., Rojas, N., Montes, M. Á. \& Climent, N. (2015). Conocimiento especializado del profesor de matemáticas (MTSK): un modelo analítico para el estudio del conocimiento del profesor de matemáticas. La Gaceta de la Real Sociedad Matemática Española, 18(3), 1801-1817.

Olarte, A. C. (2016). El auto estudio como enfoque de investigación en la formación docente. Colombian Applied Linguistics Journal, 18(2), 9-10. 
Porlán, R. (2018). Didáctica de las ciencias con conciencia. Enseñanza de las ciencias: revista de investigación y experiencias didácticas, 36(3), 5-22.

Russell, T. (2018). A teacher educator's lessons learned from reflective practice. European Journal of Teacher Education, 41(1), 4-14.

Russell, T., Fuentealba, R. \& Hirmas, C. (2016). Formadores de formadores, descubriendo la propia voz a través del self-study. Santiago: Organización de Estados Iberoamericanos. https://bit.ly/2UmR8c9

Russell, T. y Mena, J. (2016). El self-study como forma de investigación en la formación del profesorado: Un análisis de contenido de los trabajos presentados en el X Congreso Internacional sobre Self-Study S-STEP de 2014. In J. Cornejo, J. Loughran, T. Russell, A. Berry, S. Sandretto, S. Schuck, ... Mena, Formadores de Formadores, Descubriendo la Propia Voz a través del Self-Study. Santiago: BUK Grafica \& Editora.

Sanmartí, N. y Marchán, I. (2015). La educación científica del siglo XXI: retos y propuestas. Investigación y ciencia, 469, 30-38.

Santander, P. (2011). Por qué y cómo hacer Análisis de Discurso. Cinta de moebio, (41), 207-224.

Schuck, S. y Russell, T. (2016). Self-Study, amistad crítica y las complejidades en la formación de profesores. En: T. Russell, R. Fuentealba, R. \& C. Hirmas (Eds). Formadores de Formadores, descubriendo la propia voz a través del self-study. Santiago de Chile: OEI

Vallejo, R. \& Finol de Franco, M. (2009). La triangulación como procedimiento de análisis para investigaciones educativas. REDHECS, 7, 117-133.

Vanegas, C. (2016). Procesos Reflexivos de profesores de ciencias durante las prácticas pedagógicas de formación inicial (Tesis Doctoral). Santiago, Chile: Pontificia Universidad Católica de Chile.

Vanegas, C. \& Fuentealba, R. (2018). Reflexión Docente: Perspectivas Teóricas, Críticas y Modelos para el Desarrollo Profesional de Profesores. Curitiba, Brasil: Appris.

Vanegas, C. \& Fuentealba, A. (2019). Identidad profesional docente, reflexión y práctica pedagógica: Consideraciones claves para la formación de profesores. Perspectiva Educacional, 58(1), 115-138.

Veiravé, D., Ojeda, M., Núñez, C. y Delgado, P. (2006). La construcción de la identidad de los profesores de enseñanza media. Biografías de profesores. Revista Iberoamericana de Educación, 40(3), 11-32.

Vergara, C. \& Cofré, H. (2014). Conocimiento Pedagógico del Contenido: ¿el paradigma perdido en la formación inicial y continua de profesores en Chile? Estudios pedagógicos (Valdivia), 40(especial), 323-338. 


\section{Anexo 1}

\section{Categorías y subcategorías de análisis}

\begin{tabular}{|l|l|l}
\hline Tensión: Cuestionamiento de prác- & Contradicción: Situa- & Resignificación: Caracteriza- \\
ticas y supuesto que le son natu- & ciones, sentimientos o & ción y argumentación sobre \\
ralmente cómodas al didacta, que & argumentos, que son & situaciones que podrían ser \\
emergen de la atención consciente & identificados por el & enfrentadas de otras maneras o \\
de sus sentimientos, pensamientos & didacta o planteados & analizadas desde otros puntos \\
o acciones, y que derivan en nuevas & por el estudiantado, & de vista, las cuales surgen a \\
perspectivas sobre los estudiantes, el & los cuales contradicen & partir del propio análisis de \\
contexto, la didáctica específica o la & la acción docente con & las vivencias de las clases, del \\
profesionalidad. Implica el análisis & respecto a los estu- & análisis de los amigos críticos \\
de situaciones de enfrentamiento & diantes, el contexto, & o del reconocimiento de ten- \\
entre las convicciones o creencias & la didáctica específica & siones y contradicciones de la \\
personales con los planteamientos & o la profesionalidad. & práctica pedagógica. Permite \\
de los estudiantes y los elementos & Determinan desacier- & la transformación adaptativa y \\
contextuales que intervienen para & tos respecto de las & gradual, a corto o mediano pla- \\
facilitar u obstaculizar el desarrollo & formas de abordar la & zo, de cambios específicos en \\
de la docencia universitaria. Lasten- & docencia universitaria & el desarrollo de las clases o en \\
siones no generan confirmaciones o & y llevan al didacta a & las formas de comprender los \\
contradiccionesa la acción docente, & pensar que a futuro se & acontecimientos relacionados \\
sino incertidumbres o nuevos puntos & podría proceder de otra & con los estudiantes, el contex- \\
de vista sobre los acontecimientos. & manera. & to, la didáctica específica o la \\
& & profesionalidad.
\end{tabular}

Subcategorías

Estudiantes: Consideración de los acontecimientos del aula que están en relación directa con los estudiantes, así como las formas de interacción entre el didacta y los estudiantes. Esto incluye el conocimiento de sus intereses, necesidades y expectativas, así como la atención cuidadosa a sus puntos de vista y recomendaciones sobre la docencia universitaria.

Contexto: Consideración de las características generales de la población, que son heredadas por los estudiantes y profesores, condicionan el funcionamiento de la docencia universitaria y el ejercicio profesional del didacta. En particular, tiene efectos directos sobre las formas como el didacta orienta, desarrolla y aplica los contenidos específicos de la clase.

Didáctica: Reconocimiento de los niveles de coherencia entre la propia docencia universitaria y la epistemología personal que sustenta los supuestos y acciones del didacta. Esto implica, la consideración del rol del didacta y de la didáctica en la docencia universitaria, la contraposición entre los propios modelos didácticos y los acontecimientos de la sala de clase, así como el examen cuidadoso en cuanto a la congruencia entre el discurso y la acción.

Profesionalidad: Proceso dinámico e interactivo que permite configurar una representación de sí mismo como profesor universitario, movido por fases interrogativas generadas por situaciones interpelantes internas (tensiones, contradicciones o resignificaciones) y externas (el contexto, los estudiantes o los amigos críticos). Implica la consideración de las características y capacidades específicas del didacta en su rol de formador, el cual lo faculta para tensionar las perspectivas y expectativas profesionales de sus estudiantes, así como la visión, sentido e identidad profesional. 